\title{
IPSF: análise da estrutura interna em uma amostra de jovens adultos portugueses
}

\author{
Marta Gonçalves ${ }^{1}$ \\ Instituto Universitário de Lisboa (ISCTE-IUL), Cis-IUL, Lisboa, Portugal \\ Makilim Nunes Baptista \\ Universidade de São Francisco, Itatiba-SP, Brasil \\ Diana Farcas \\ Instituto Universitário de Lisboa (ISCTE-IUL), Cis-IUL, Lisboa, Portugal
}

\section{RESUMO}

A avaliação da percepção que se tem da família é fundamental para saber quanto os jovens estão satisfeitos com essa instituição social, sendo que em Portugal não existe uma escala com essa função. O objetivo deste estudo foi avaliar as semelhanças psicométricas do conjunto inicial de 136 itens que foi utilizado na construção do Inventário de Percepção do Suporte Familiar (IPSF) no Brasil, tendo sua versão final com 42 itens e comparar os resultados em uma amostra portuguesa de jovens adultos $(N=248)$. Para o tratamento dos dados, recorreu-se à mesma análise realizada no Brasil, de componentes principais com rotação Oblimin. Os resultados demonstraram um número de itens e distribuição nas três categorias iniciais (afetivo-consistente, adaptação e autonomia) muito próximos aos encontrados na amostra de normatização brasileira, o que sugere semelhança tanto no número quanto na constituição dos componentes nas duas culturas.

Palavras-chave: família; testes psicológicos; psicometria.

\section{ABSTRACT - IPSF: Analysis of internal structure in a sample of Portuguese young adults}

The evaluation of the people's perception of family is fundamental to understanding the degree to which young people are satisfied with this social institution, and in Portugal there is no scale with this function. The objective of this study was to evaluate the psychometric similarities of the initial set of 136 items that was used in the construction of Family Support Perception Inventory (IPSF) in Brazil, having a final version with 42 items, and compare the results to a Portuguese sample of young adults $(N=248)$. For the data processing, we used the same analysis as in Brazil, of the principal components with oblimin rotation. Results showed a number of items and distribution in the three initial categories (affective-consistent, adaptation and autonomy) very close to those found on the Brazilian standardization sample, suggesting similarity both in number and in the constitution of the components in the two cultures.

Keywords: family; psychological testing; psychometrics.

RESUMEN - IPSF: análisis de la estructura interna en una muestra de adultos jóvenes portugueses

La evaluación de la percepción que se tiene sobre la familia es fundamental para saber en qué medida los jóvenes están satisfechos con esta institución social; en Portugal no existe una escala con esta función. El objetivo de este estudio fue evaluar las semejanzas psicométricas del conjunto inicial de 136 ítems que se utilizan en la construcción del Inventario de Percepción de Soporte Familiar (IPSF) en Brasil, teniendo su versión final con 42 ítems, y comparar los resultados en una muestra portuguesa de jóvenes adultos $(N=248)$. Para el tratamiento de los datos se recurrió al mismo análisis realizado en Brasil, de componentes principales con rotación oblimin. Los resultados mostraron una serie de ítems y distribución en las tres categorías iniciales (afectivo-consistente, adaptación y autonomía) muy cercanos a los encontrados en la muestra brasileña de normalización, lo que sugiere semejanzas tanto en número como en la constitución de los componentes en las dos culturas.

Palabras clave: familia, tests psicológicos, psicometría.

Numa altura em que as famílias portuguesas enfrentam novas pressões e desafios no seu cotidiano resultantes de transformações socioculturais e econômicas vividas recentemente na Europa, torna-se essencial estar, enquanto profissional, muito bem preparado para apoiá-las. Essas pressões e desafios afetam negativamente a sua saúde mental, bem-estar e qualidade de vida. São elas uma maior taxa de divórcio, uma menor taxa de 
casamento, o emprego limitado, o aumento da mobilidade e da competição, o ritmo acelerado, mais dificuldades nas relações interpessoais, atividades de lazer mais passivas, taxas mais elevadas de obesidade, entre outros (Gonçalves, Farcas, \& Cook, 2014). Todo esse estresse compromete o atual e futuro desenvolvimento dos seus membros. Um dos aspectos que pode fazer a diferença enquanto profissional que apoia e estuda as famílias é conhecer o suporte familiar. O suporte familiar recebido e percebido, prestado pela família em situações comuns da vida cotidiana e/ou em momentos de crise em que existe maior vulnerabilidade ou risco pode ser considerado um importante amortecedor de estressores na vida do indivíduo. Contudo, muitos dos profissionais que acompanham as famílias portuguesas, principalmente as mais carenciadas, resistem à adoção de uma avaliação e intervenção mais positiva e socioecológica, devido a pressões burocráticas promovidas pelas políticas sociais em vigor (Golçalves, Farcas, \& Martins, 2013).

Se por um lado os pais de jovens adultos (18-30 anos) continuam a oferecer suporte/apoio emocional aos filhos durante a sua idade adulta (Fingerman, Miller, Birditt, \& Zarit, 2009), por outro também os filhos apoiam os pais (Buhl, 2009). E parece que ambos oferecem mais suporte quando o outro se encontra em necessidade (Fingerman et al., 2009). O suporte familiar é um dos fatores protetores mais importantes, atenuando o efeito de múltiplos estressores na vida das pessoas (Baptista, 2005). O construto avalia, entre outros, a percepção de afetividade entre os membros da família, comunicação, regras, sentimento de pertença; e é baseado na teoria de Olson, Russel, e Sprenkle (1983) que desenvolveram um modelo circumplexo de famílias. O suporte familiar adequado já foi associado a diversos construtos, como maior saúde mental e adesão a tratamento retroantiviral (Camargo, Capitão, \& Filipe, 2014), menos estresse e depressão (Alves, Baptista, \& Santos, 2014; Camargo, Calais, \& Sartori, 2015), menos desesperança e depressão (Baptista, Carneiro, \& Cardoso, 2014), menos comportamentos aditivos (Baptista, Lemos, Carneiro, \& Morais, 2013), menos comportamentos de risco e menor saúde mental (Souza, Baptista, \& Baptista, 2010), entre outros. Os adolescentes e jovens adultos que percepcionam a sua família como apoiante relatam um maior nível de satisfação com a vida (Pardeck el al., 1991). Do mesmo modo, Ross e Mirowsky (2002) indicam que uma percepção adequada do suporte familiar está relacionada com o aumento do sentimento de segurança.

Baptista (2005) conduziu uma revisão de instrumentos que avaliassem a percepção do suporte familiar no Brasil e constatou que existem instrumentos que focam alguns aspectos do funcionamento familiar, nomeadamente a Entrevista Familiar Estruturada (Carneiro, 1983), o Questionário de Percepção dos Pais (Pasquali \& Araújo, 1986), o Inventário de Estilos Parentais
(Gomide, 2003) e o Inventário de Práticas Parentais (Benetti \& Balbinotti, 2003). Todos esses instrumentos focam a avaliação da relação dos pais com os filhos, e não a percepção do suporte familiar, independentemente da faixa etária. No sentido de ultrapassar essa lacuna, Baptista $(2005$; 2007) desenvolveu e validou, no Brasil, uma escala para avaliar a percepção do suporte familiar, que continha inicialmente 136 itens, construídos com base num estudo brasileiro e em diversos instrumentos de avaliação familiar.

Após análises psicométricas iniciais com os 136 itens, a escala final ficou com 42 itens e denomina-se Inventário de Percepção do Suporte Familiar (IPSF), avaliando três fatores: afetivo-consistente, adaptação e autonomia (Baptista, 2009). O IPSF foi aplicado em diferentes amostras brasileiras, utilizando a Teoria Clássica e a Teoria de Resposta ao Item (TRI). Num dos estudos iniciais com 1064 estudantes do ensino médio e universitário, procedeu-se a uma análise fatorial por componentes principais, com cargas fatoriais acima de 0,30 e com rotação oblimin. Os resultados apontaram para uma configuração de 42 itens distribuídos em três dimensões já relatadas anteriormente, com variância explicada de 41,43\% (Baptista, 2007). Gomes e Baptista (2013) analisaram o IPSF utilizando a TRI e numa amostra de 1322 participantes $(M=22,79$ anos; $D P=5,25)$ encontraram índices adequados dos itens a partir da análise dos infits $(M=1,01 ; D P=0,24)$ e distribuição das dificuldades dos itens de forma esperada no mapa de itens, variando de pouco a muito. Além desses resultados, encontrou-se funcionamento diferencial de itens para três, favorecendo o público feminino e quatro itens favorecendo o masculino, ou seja, equilíbrio em ambos os sexos.

Além de validade da estrutura interna e baseada na relação com outras variáveis, Capitão, Baptista, Marques e Meireles (2013) encontraram, no Brasil, evidências de validade com grupos critério, especificamente com três grupos: um de 102 pacientes atendidos num serviço de atendimento psicoterapêutico para problemas familiares, um grupo de 344 acompanhantes e um terceiro grupo de 603 universitários sem queixas de problemas familiares. Os resultados demonstraram diferenças significativas entre os grupos nos três fatores, a partir de um modelo de efeito de interações com faixas etárias, escolaridade e características dos grupos, sendo que esta última variável é a que explica melhor a diferença das pontuações.

Em Portugal, existem duas escalas validadas para a população portuguesa no âmbito do suporte social. A Escala Multidimensional de Suporte Social Percebido (MSPSS) de Carvalho, Pinto-Gouveia, Pimentel, Maia, e Mota-Pereira (2011) e a Escala Comportamental de Suporte Social Familiar para Adolescentes com Diabetes (DFBS) de Almeida e Pereira (2011). Enquanto a primeira avalia subjetivamente o suporte 
social proveniente da família, dos amigos e de outros significativos, a segunda avalia duas áreas do apoio familiar relevantes para ajudar a criança ou o adolescente a lidar com as exigências da diabetes e do tratamento: controle-aconselhamento e afeto.

Contudo, uma vez que essas escalas se destinam a públicos e/ou contextos diferentes e específicos, quando se compara as características do IPSF com esses dois instrumentos adaptados para Portugal, do ponto de vista geral, as três escalas diferem em termos de variância explicada, número de fatores e itens, teoria de base, tipos de resposta requerida e até mesmo objetivos propostos. Por exemplo, a DFBS possui características bastante parecidas com o IPSF. Em termos de número de itens, a DFBS é ligeiramente menor, possui variância explicada semelhante ao IPSF, é constituída por dois componentes - controle/aconselhamento e afetivo, com fiabilidade de cada dimensão menor que o IPSF, mas é destinada especificamente a um tipo de amostra constituída por adolescentes com diabetes. A DFBS possui essa característica de estar destinada a essa amostra com características próprias relacionadas a um tipo de doença e a uma faixa etária, provavelmente não sendo recomendada para uma utilização mais geral. A DFBS cumpre bem, do ponto de vista psicométrico, os seus objetivos e pode ser bastante útil no diagnóstico familiar e intervenção nesses casos de doença física. Já a MSPSS é uma escala com um número de itens bastante menor que o IPSF, que abrange o suporte social geral (familiar e amigos), com uma variância explicada muito considerável e que pode ser utilizada de forma geral, provavelmente mais aconselhada para um rastreio e/ou uma análise mais global do suporte, cumprindo muito bem esse papel.

Nos casos em que o investigador ou o profissional de saúde pretende um panorama rápido desse construto combinado (suporte proveniente de amigos e da família), a MSPSS pode ser bastante indicada. Um aprofundamento de um ou outro requer, contudo, uma escala que ofereça maiores detalhes, já que existem escalas inteiras para avaliar apenas o suporte social ou apenas o suporte familiar. O IPSF é uma opção para o investigador ou o profissional de saúde que pretende um aprofundamento de informações mais completas a respeito apenas do suporte familiar. O IPSF possui características psicométricas adequadas, foi desenvolvido numa cultura bastante próxima à portuguesa, inclusive com uma linguagem também muito próxima semanticamente, e pode ser utilizado em diversos contextos por não ser destinado a um tipo único de amostra.

Além disso, pode oferecer informações bastante detalhadas sobre a percepção que a pessoa possui sobre as questões afetivas, as regras, os sentimentos dentro da família, a comunicação familiar, as relações familiares, a resolução de problemas, a proximidade entre os membros, o respeito, entre outras características contempladas qualitativamente em cada um dos itens das três dimensões.
Por exemplo, um profissional de saúde pode fazer uma avaliação mais minuciosa e, a partir desses dados, planear intervenções mais voltadas para cada uma dessas características. Por outro lado, um investigador social pode ter um diagnóstico das características mais acentuadas num grupo específico.

Independentemente da utilidade de um instrumento psicológico na prática de profissionais de saúde, é importante que ele possua características psicométricas adequadas ao seu uso. Para além disso, como apontam Bollen e Diamantopoulos (no prelo), indicadores reflexivos dependeriam de uma variável latente que explicaria o fenômeno e haveria uma tendência de alguns construtos serem estáveis em diferentes locais.

Um instrumento psicológico pode ser construído de diversas maneiras, mas tradicionalmente seguem-se alguns passos. Como aponta Adanéz (1999), as etapas que constituem a construção de um teste psicológico envolvem a definição dos objetivos do teste, especificação do contexto, modelo estatístico, a definição da teoria, construção dos itens e das instruções, seleção das amostras, aplicação inicial, análise e seleção empírica dos itens, avaliação da precisão e da validade do teste, entre outras.

Assim sendo, quando um teste é concebido, geralmente o autor, baseado na teoria de base, desenvolve um número maior de itens, que, a partir de análises de conteúdo e principalmente das análises empíricas estatísticas, serão escolhidos aqueles que mais discriminam o fenômeno estudado do ponto de vista de sua estrutura interna. Esse processo é diferente da adaptação e consequente validação de instrumentos entre culturas diferentes, que também possui passos fundamentais para a garantia das propriedades psicométricas (Borsa, Damásio, \& Bandeira, 2012), mas que, nesses casos, o instrumento já vem com esses melhores itens escolhidos. S e g u n d o Brown (2006), uma das aplicações de um conjunto de procedimentos estatísticos denominados de análise exploratória é a confirmação ou refutação de uma estrutura fatorial de determinado instrumento. Uma vez que em Portugal não foi possível encontrar uma escala que avaliasse especificamente a percepção do suporte familiar como o ISPF o faz, o objetivo deste trabalho foi o de analisar os 136 itens iniciais do desenvolvimento do IPSF brasileiro (Baptista, 2005), mas agora em uma amostra de portugueses, utilizando o mesmo método de extração dos fatores, bem como a mesma rotação nos itens originais, no sentido de avaliar se os mesmos itens que resultaram na escala final de 42 itens finais do IPSF do Brasil também iriam ser similares àqueles da amostra portuguesa, além de poder trazer para Portugal uma escala que avaliasse a percepção do suporte familiar. No entanto, foi apenas testado o instrumento final com 42 itens, traduzidos e adaptados para o português de Portugal. Nunca se fez um estudo envolvendo os 136 itens iniciais do IPSF em Portugal. 


\section{Método}

\section{Participantes}

Neste estudo participaram 248 jovens adultos com uma média de idade de 20,8 anos ( $D P=9,1$ anos), de uma amostra de conveniência em que o único critério de inclusão era a idade ser entre 18 e 30 anos. A razão de optar por jovens adultos prendeu-se com o fato de ser uma população mais acessível. A maioria desses jovens era de nacionalidade portuguesa $(93,6 \%)$, do sexo feminino (72,3\%) e solteiros (93,6\%). A razão de incluir 6,3\% de participantes estrangeiros na adaptação portuguesa deveu-se ao fato de se poder ter um instrumento adaptado para Portugal que possa ser passado a estrangeiros no país desde que dominem a língua. Mais de metade desses jovens eram estudantes $(69,1 \%)$, tendo concluído o ensino médio (52,2\%). A maioria residia no distrito de Lisboa $(80,6 \%)$ com a mãe $(81,9 \%)$, o pai $(65,9 \%)$ e os irmãos (50,6\%). Menos de metade dos jovens (40,6\%) afirmava que o rendimento mensal do agregado familiar se situava entre os 1000 e 2000 euros.

\section{Instrumento}

$\mathrm{O}$ instrumento utilizado foram os 136 itens iniciais do IPSF (ex.: "planear atividades em família é difícil”; “a minha família tenta mimar-me"; "gosto de estar com a minha família”) e pedia-se aos participantes para indicar a frequência com que consideravam que essas afirmações aconteciam na sua família, pensando na sua família de origem (pais, irmãos, avós), utilizando a escala de resposta de quatro pontos $(1=$ sempre; $2=$ muitas vezes; $3=$ poucas vezes; $4=$ nunca). Esses itens foram inicialmente aplicados numa amostra de mais de 1000 participantes no Brasil e adaptados para Portugal por um processo completo de adaptação cultural de itens com tradução, retrotradução, comparação das versões e aplicação piloto com modificações de palavras e expressões, ao contrário dos primeiros estudos de adaptação de instrumentos que se restringiam apenas à tradução (Reichenheim \& Moraes, 2007). O objetivo desse processo é que o conteúdo do IPSF se mantivesse, mas que pudesse ser facilmente compreendido na cultura portuguesa. Assim sendo, a versão do IPSF para português de Portugal foi desenvolvida de acordo com as seguintes fases: tradução, retrotradução, comparação e aplicação-piloto.

$\mathrm{O}$ processo de tradução foi inicialmente realizado por uma pessoa com nacionalidade portuguesa e com formação em Psicologia. Pediu-se que procedesse a leitura de cada item e, em seguida, indicasse quais as alterações semânticas (significado da palavra) e idiomáticas (uso de expressões) necessárias a serem realizadas para que o item fosse escrito em português de Portugal. Em seguida, na fase da retrotradução, isto é, a tradução dos itens para o idioma original, participaram duas pessoas de nacionalidade brasileira com formação em Psicologia. A tarefa delas consistiu em, individualmente, alterar cada um dos itens apresentados em português de Portugal para português do Brasil. As duas versões emergentes da retrotradução foram comparadas com a versão original, por quatro investigadores com nacionalidade portuguesa e com formação em Psicologia. Após a avaliação individual, os investigadores reuniram-se e discutiram as conclusões que retiraram das duas versões termos semânticos e idiomáticos. Tendo em conta que se verificaram poucas mudanças no IPSF, uma versão de consenso foi facilmente atingida nessa fase de comparação da adaptação cultural. Após essa fase, procedeu-se a aplicação-piloto do IPSF. Assim sendo, o IPSF foi aplicado a quatro pessoas com idade entre os 20 e 22 anos, que foram esclarecidas sobre a finalidade da sua participação e indicou-se que deveriam expressar a sua opinião relativamente à clareza e compreensão dos itens, bem como o preenchimento do questionário (Baptista, Lemos et al., 2013).

Inventário de Percepção do Suporte Familiar - IPSF (Baptista, 2009). Os itens do Inventário de Percepção de Suporte Familiar (IPSF) foram construídos com base no modelo teórico de Olson et al. (1983), tendo como base diversos instrumentos nacionais e internacionais, tais como o Family Adaptability and Cohesion Evaluation Scale (FACES-III) de Olson, Portner, e Lavee (1985); o Family Assessment Device (FAD) de Epstein, Baldwin, e Bishop (1983); o Family Awareness Scale (FAZ) de Green, Kolevzo e Vosler (1985); o Parental Bonding Instrument (PBI) de Parker, Tupling, e Brown (1979) e o Questionário de Estilos Parentais de (Gomide, 2013). Além dessa base teórica e instrumental, foi realizado um estudo com 100 estudantes universitários de Psicologia de uma universidade do interior de São Paulo com a pergunta aberta "Na sua opinião, o que é uma família ideal?". Os resultados foram analisados em termos de conteúdo e os itens construídos também a partir dessas respostas (Baptista, 2005). A análise da estrutura interna no Brasil contou com uma amostra de 1064 estudantes $(M=23,4$ anos; $D P=6,1)$ do ensino público e privado do estado de São Paulo. Após a análise fatorial exploratória com o método de componentes principais e rotação oblimin, o IPSF brasileiro ficou composto por 42 itens que avaliam como a pessoa percebe o suporte que recebe da sua família. É uma escala Likert de três pontos, com variação de zero a dois (quase nunca ou nun$\mathrm{ca}=0$; às vezes $=1$; quase sempre ou sempre $=2$ ), e com pontuação mínima de zero e máxima de 84 pontos. $\mathrm{O}$ inventário é dividido em três dimensões, sendo estas: 1. Afetivo-consistente, composta por 21 itens sobre relações afetivas positivas, carinho, proximidade, clareza de papéis e regras dos membros da família e competência na resolução de problemas; 2. Adaptação com 13 itens sobre sentimentos negativos sobre a família, tais como raiva, isolamento, exclusão, vergonha, incompreensão e interesse, sendo que esse fator deve ser pontuado inversamente; e 3. Autonomia com oito itens sobre relações 
de confiança, liberdade e privacidade. Os índices de fiabilidade baseados no alfa de Cronbach demonstraram que a dimensão Afetivo-consistente apresentou um alfa de 0,91 ; a dimensão Adaptação, um alfa de 0,90 ; a dimensão Autonomia, um alfa 0,78, além do valor de 0,93 para o inventário total. Quanto maior a pontuação no instrumento, melhor o suporte familiar percebido pela pessoa. No Brasil, com a amostra total, foram calculados os níveis em que é possível alocar os participantes nas normas, sendo dividido em quatro possibilidades, tanto nas dimensões quanto na pontuação geral, ou seja, o participante pode ser alocado nos níveis: baixo, médio-baixo, médio-alto e alto de avaliação da sua percepção do suporte familiar.

\section{Procedimento}

Os dados foram recolhidos utilizando as duas versões do questionário, online $(n=106)$ e em papel $(n=142)$. Numa primeira fase, utilizou-se o questionário online disponível na plataforma do Qualtrics e distribuiu-se o link por contatos pessoais, redes sociais e por meio da lista de e-mails dos alunos de várias universidades portuguesas públicas e privadas. Para aumentar a amostra, deu-se início à segunda fase de recolha utilizando o questionário em papel, distribuídos por uma amostra por conveniência de docentes de diferentes áreas (Psicologia, Sociologia, Gestão, Finanças) da universidade a que o estudo se encontra associado e da qual recebeu aprovação, por meio do Comitê de Ética Institucional do Centro de Investigação e Intervenção Social. Quer na versão online quer em papel, todo o rigor ético e de anonimato foi garantido de acordo com as normas internacionais (APA, 2002; EFPA, 2005) e nacionais (OPP, 2011). A duração média do preenchimento do questionário foi de aproximadamente 20 minutos, e a recolha dos dados em papel e online decorreu entre julho de 2012 e maio de 2013.

\section{Análise de Dados}

Os dados foram analisados utilizando o software SPSS versão 18. Realizou-se inicialmente uma análise exploratória em componentes principais (ACP) com rotação oblimin, com o objetivo de determinar o número de componentes significantes (valores próprios maiores que 1. produzidos pelos itens. A utilização do gráfico de sedimentação, mesmo não sendo um método unânime entre psicometristas, nesse caso pôde ser bem empregue, já que os fatores estavam bem definidos e a sua utilização foi feita para a confirmação de uma estrutura fatorial anterior (Damásio, 2012). Posteriormente, foi conduzida uma nova ACP solicitando a extração de três fatores, um peso (loading factor) superior a $|0,40|$ e rotação oblimin, conforme a análise original do IPSF (dos 136 itens iniciais), no entanto, aumentando de 0,35 para 0,40 o valor de supressão da carga fatorial. A utilização do ponto de supressão dos itens maior na amostra de Portugal também se deu pela aproximação do número de itens das duas amostras, com o aumento do valor, obtendo-se então um instrumento muito parecido com o original. Foram também realizadas as análises de fiabilidade na escala geral e em cada dimensão, utilizando-se o alfa de Cronbach.

\section{Resultados}

Os resultados da ACP com rotação oblimin apontaram para uma adequabilidade muito boa $(\mathrm{KMO}=0,949$; $\left.\chi^{2}=6794,54 ; p<0,001\right)$ e sugeriram a extração de seis componentes que explicavam cerca de $49,36 \%$ da variância global. Simultaneamente, uma inspeção ao gráfico de sedimentação, apresentado na Figura 1, revelou que. a partir da terceira componente formava-se uma linha horizontal, indicando a possibilidade de a variância única dominar a estrutura da variância comum (Hair, Black, Babin, \& Anderson, 2010), sugerindo a existência de três a quatro componentes. No entanto, como o objetivo era de avaliar a comparação entre as duas culturas, na análise final, foram impostos os três componentes da escala original IPSF (Baptista, 2005). Além disso, um item não carregou quando se utilizou o ponto de corte de 0,35 , sugerindo também a retirada precoce dele da análise seguinte.

Os resultados da segunda ACP indicaram uma adequabilidade muito boa $(\mathrm{KMO}=0,942)$, com uma variância explicada de 31,91\% (com eigenvalues dos três fatores de 16,2; 3,9 e 2,8). Em sete interações na rotação, o primeiro fator explica $12,84 \%$; o segundo $10,74 \%$ e o terceiro 8,33\%. A distribuição dos itens na análise fatorial encontra-se na Tabela 1.

Da mesma forma que o original, as dimensões mantiveram-se bastante estáveis, ocorrendo, como referido anteriormente, a retirada de um item e a migração de um item para a primeira dimensão. A primeira dimensão, contendo 22 itens manteve a nomenclatura original de Afetivo-consistente, com itens sobre relações afetivas positivas, carinho, proximidade, clareza em papéis e regras dos integrantes da família e habilidade na resolução de problemas. A segunda dimensão, denominada de Adaptação, ficou com 11 itens sobre sentimentos negativos sobre a família, tais como raiva, isolamento, exclusão, vergonha, incompreensão e interesse. E, por último, a dimensão Autonomia, com oito itens sobre relações de confiança, liberdade e privacidade.

O alfa do IPSF como um todo foi de 0,86 e para as dimensões os resultados distribuíram-se da seguinte forma: Afetivo-consistente $(\alpha=0,95)$; Adaptação $(\alpha=0,92)$ e Autonomia $(\alpha=0,87)$. As correlações entre as dimensões foram: Afetivo-consistente com Adaptação $(r=-0,40)$; Afetivo-consistente com Autonomia $(r=0,35)$; Adaptação com Autonomia $(r=-0,40)$. Os resultados negativos dão-se devido à segunda dimensão se tratar de itens que possuem o sentido negativo, diferente das dimensões 1 e 3 . 


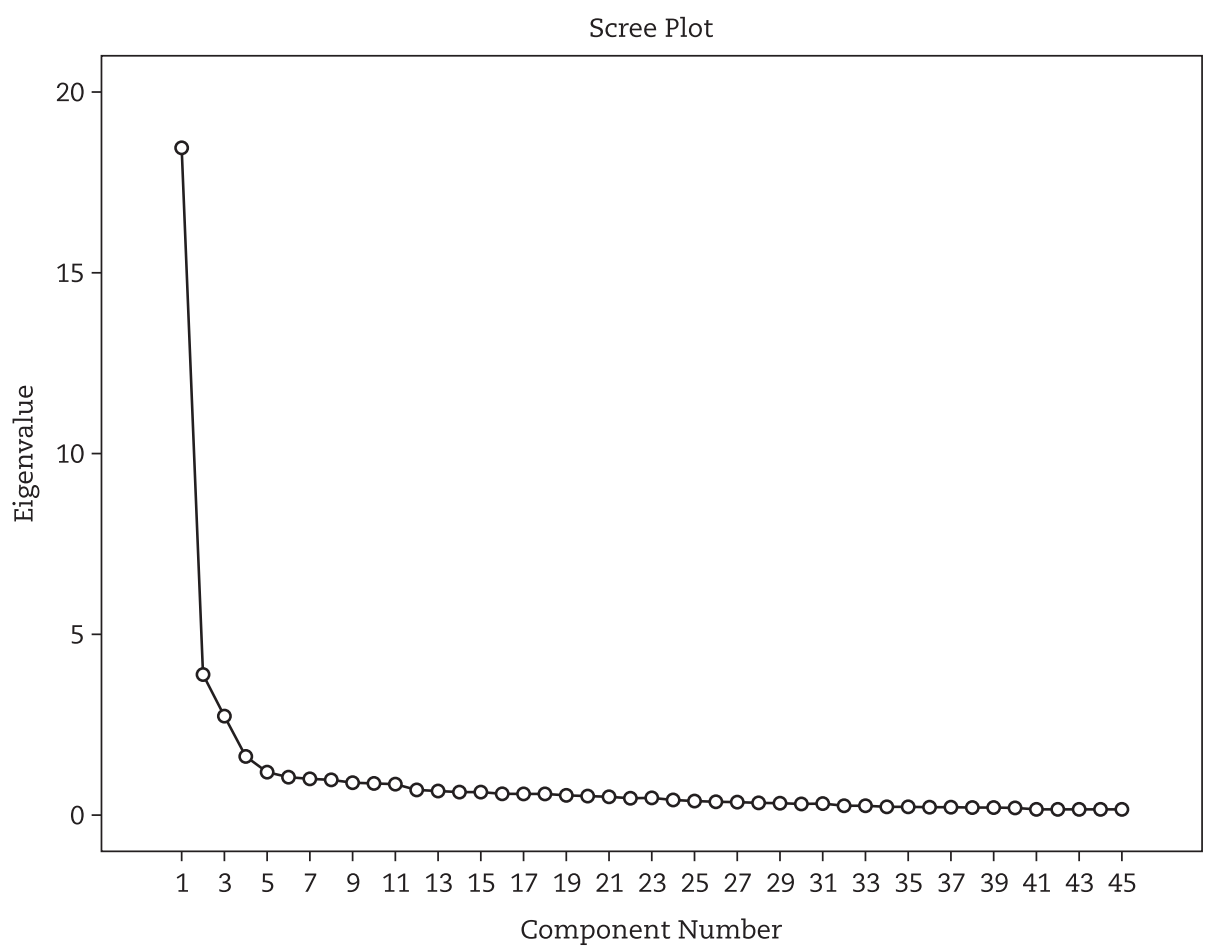

Figura 1. Gráfico de sedimentação

Tabela 1

Análise Factorial do IPSF

\begin{tabular}{ll}
\hline & \multicolumn{1}{c}{ Componentes } \\
\cline { 2 - 2 } Na minha família, conversamos sobre planos familiares. & 1 \\
A minha família expressa claramente pensamentos e emoções entre si. & 0,77 \\
Na resolução de problemas, temos em conta a opinião de toda a família. & 0,76 \\
Na minha família, diz-se o que se sente uns pelos outros. & 0,73 \\
Na minha família, as tarefas são distribuídas adequadamente. & 0,72 \\
Na minha família, expressamos interesse uns pelos outros. & 0,70 \\
Há regras para diversas situações na minha família. & 0,69 \\
A minha família tenta pensar sobre diferentes maneiras de resolver os problemas. & 0,68 \\
As pessoas da minha família gostam de passar o tempo juntas. & 0,68 \\
A minha família segue as regras por si estabelecidas. & 0,67 \\
Cada um, na minha família, tem deveres e responsabilidades específicas. & 0,66 \\
Eu e a minha família conversamos sobre coisas de interesse para todos. & 0,64 \\
Na minha família, as pessoas tocam-se e abraçam-se. & 0,63 \\
A minha família partilha os seus sentimentos. & 0,62 \\
A minha família faz-me sentir melhor quando estou aborrecido (a). & 0,62 \\
A minha família sabe o que fazer quando há uma emergência. & 0,62 \\
Na minha família. pode-se falar uns com os outros sobre a tristeza que se sente. & 0,56 \\
A minha família elogia-me. & 0,54 \\
A minha família sabe quando alguma coisa má me aconteceu, mesmo que eu não diga. & 0,50 \\
A minha família proporciona-me conforto emocional. & 0,47 \\
A minha família sente-se mais próxima entre si do que com pessoas de fora. & 0,47 \\
A minha família é respeitada por aqueles que a conhecem. & 0,47 \\
Sinto raiva da minha família. & 0,42 \\
Sinto vergonha da minha família. & \\
\hline & 0,88 \\
& 0,85 \\
\hline
\end{tabular}




\begin{tabular}{lc}
\hline & Componentes \\
\cline { 2 - 2 } Sinto-me excluído (a) da minha família. & 1 \\
Os elementos da minha família só pensam em si próprios. & 0,83 \\
Sinto-me como um (a) estranho (a) na minha família. & 0,76 \\
Há ódio na minha família. & 0,76 \\
Na minha família, só se mostra interesse uns pelos outros quando se pode ter vantagens. & 0,75 \\
Parece haver uma série de atritos na minha família. & 0,72 \\
A minha família irrita-me. & 0,71 \\
Acredito que a minha família tem mais problemas emocionais do que as outras famílias. & 0,68 \\
Na minha família, culpa-se alguém quando as coisas não correm bem. & 0,64 \\
A minha família deixa-me ser como quero. & 0,59 \\
Na minha família, é permitido que eu faça o que gosto. & 0,82 \\
A minha família deixa-me decidir o que quero. & 0,79 \\
A minha família deixa-me sair tanto quanto quero. & 0,78 \\
A minha família aceita-me como sou. & 0,76 \\
A minha família faz-me sentir capaz de cuidar de mim, mesmo quando estou sozinho (a). & 0,60 \\
A minha família concorda com o meu estilo de vida. & 0,52 \\
Na minha família, tenho privacidade. & 0,45 \\
\hline
\end{tabular}

\section{Discussão}

O objetivo deste trabalho foi de replicar a análise inicial com os 136 itens de construção do IPSF e avaliar como esses itens iriam se comportar numa amostra portuguesa, no intuito de se avaliar se a mesma configuração e itens ocorreriam ou se outros itens surgiriam no contexto português. Portanto, o objetivo consistiu em utilizar o mesmo método e rotação do estudo brasileiro no sentido de comparar os resultados e poder trazer para Portugal uma escala que avaliasse a percepção do suporte familiar.

Um estudo intercultural anterior utilizou o IPSF numa amostra de 280 adolescentes portugueses e brasileiros, com o objetivo de se avaliar o funcionamento diferencial dos itens (DIF) de respostas entre as duas culturas por intermédio da análise da Teoria de Resposta ao Item (TRI). Os resultados apontaram para a existência de bons índices de ajustes dos itens em Portugal, indicando que existem poucas diferenças nas respostas dos participantes das duas culturas (Baptista, Gonçalves, Carneiro, \& Silva, 2013). No entanto, é importante referir que o objetivo desse estudo não incidiu sobre a avaliação da estrutura interna do instrumento em termos de fatores e número de itens finais nos dois países.

Após a adaptação semântica dos itens iniciais, quase a totalidade desses e dos fatores iniciais permaneceram na escala final, o que demonstra que há um padrão de resposta muito semelhante nas duas culturas. As análises iniciais revelaram que os dados encontrados cumprem com a maioria dos requisitos estabelecidos para a condução da análise de componentes principais. Sobre a natureza das variáveis, vários autores (e.g., Hair et al., 2010) referem que as variáveis de input (itens) devem ser variáveis métricas ou tratadas como tal. Nesse caso específico, as variáveis de input foram tratadas como se fossem métricas, visto que foram apresentadas numa escala Likert de quatro pontos.

Já que, na psicometria, a análise de fatores possui particular relevância para a estrutura interna de uma variável latente (Dancey \& Reidy, 2006), pode-se afirmar que o IPSF, independentemente da cultura, apresentou estabilidade fatorial. Os valores do KMO foram excelentes, além de apresentar variância um pouco menor na amostra portuguesa, o que pode ser hipotetizado pelo tamanho da amostra e estilo de resposta da amostra portuguesa e a maior ou menor discriminação de determinados itens nas duas amostras. Isso deve ser avaliado em estudos futuros a partir de amostras maiores e com recurso à análise confirmatória. Uma análise confirmatória será mais adequada a partir dos próximos estudos quando o instrumento final português for aplicado com os seus 41 itens finais. A fiabilidade dos fatores individuais foi maior que no estudo original brasileiro (Baptista, 2007). Como as cargas fatoriais foram aumentadas em relação ao estudo brasileiro, nota-se que os itens finais da escala portuguesa se encontram mais representativos do construto.

Por ser um construto que está diretamente associado à saúde mental e a fenômenos como o estresse, a depressão, a desesperança e comportamentos de risco, a possibilidade de se ter um instrumento que avalia o suporte familiar pode ser bastante útil para os clínicos e investigadores na área da saúde. Sendo assim, se detectada a percepção de baixo suporte familiar, pode-se pensar em 
intervenções sociais/familiares que sirvam para os membros se sentirem mais queridos, com maior possibilidade de serem apoiados em diversas situações, como também com maior possibilidade de terem uma comunicação mais efetiva para enfrentar as problemáticas do cotidiano, auxiliando assim os membros na sensação de pertença a um grupo social, acolhimento e servindo como um amortecedor e/ou fator protetivo da saúde mental.

Em termos de conclusão, a utilização de um mesmo instrumento por diversas culturas permite comparações de amostras e contextos que ampliam o conhecimento sobre o fenômeno em estudo. Contudo, mesmo com os resultados encontrados, alguma cautela deve ser apontada quando se pensa no tamanho e composição da amostra do presente estudo. Estudos futuros, com amostras maiores e mais variadas, poderão gerar a possibilidade de normas específicas para Portugal, já que não é adequado a sobreposição das normas de um país para outro, por mais que os instrumentos possam apresentar-se semelhantes, tanto em número de itens quanto na semântica e distribuição nas dimensões.

Um instrumento de percepção do suporte familiar pode ser bastante útil em diversos contextos, especificamente naqueles associados à saúde mental, já que essa variável se mostra um importante fator de prevenção no desenvolvimento de diversos transtornos. O ISPF pode ser utilizado como um importante aliado tanto na atenção primária à saúde como em intervenções mais complexas que envolvam o auxílio da família na recuperação de problemas de saúde física e mental.

\section{Referências}

Adánez, G. P. (1999). Procedimientos de construccion y analisis de tests psicometricos. Em S. M. Wechsler \& R. S. L. Guzzo (Eds.). Avaliação psicológica: perspectiva internacional (pp. 57-100). São Paulo: Casa do Psicólogo.

Almeida, P., \& Pereira, M. G. (2011). Escala comportamental de suporte social familiar para adolescentes com diabetes (DFBS). Psicologia, Saúde \& Doenças, 12(1), 55-75.

American Psychological Association [APA]. (2002). Ethical principles of psychologists and code of conduct. Recuperado de https://www.apa.org/ ethics/code/principles.pdf

Alves, G. A. S., Baptista, M. N., \& Santos, A. A. A. (2014). Relation between depression, family support and stress at work in undergraduates. International Journal of Psychology and Behavioral Sciences, 4(1), 9-15. doi:10.5923/j.ijpbs.20140401.02

Baptista, M. N. (2005). Desenvolvimento do inventário de percepção de suporte familiar (IPSF): estudos psicométricos preliminares. Psico-USF, 10(1), 11-19.

Baptista M. N. (2007). Inventário de percepção de suporte familiar (IPSF): estudo componencial em duas configurações. Psicologia: Ciência e Profissão, 27(3), 496-509.

Baptista, M. N. (2009). Inventário de percepção do suporte familiar (IPSF). São Paulo: Vetor.

Baptista, M. N., Lemos, V. A., Carneiro, A.M., \& Morais, P. R. (2013). Perception of family support in dependents of alcohol. Adicciones (Palma de Mallorca), 25(3), 220-225.

Baptista, M. N., Gonçalves, M., Carneiro, A. M., \& Silva, J. (2013). Perception of family support in brazilian and portuguese families: An intercultural study on item structure and differential functioning. Revista Transcultural, 1(1), 95-112.

Baptista, M. N., Carneiro, A. M., \& Cardoso H. F. (2014). Depression, family support and hopelessness: A correlated study. Universitas Psychologica, 13(2), 693-702. doi: 10.11144/Javeriana.UPSY13-2.dfsh

Benetti, S. P. C., \& Balbinotti, M. A. A. (2003). Elaboração e estudo de propriedades psicométricas do inventário de práticas parentais. Psico-USF, 8(2), 103-113

Bollen, K. A., \& Diamantopoulos, A. (no prelo). In defense of causal-formative indicators: A minority report. Psychological Methods. Recuperado de http://www.ncbi.nlm.nih.gov/pubmed/26390170

Borsa, J. C., Damásio, B. F., \& Bandeira, D. R. (2012). Adaptação e validação de instrumentos psicológicos entre culturas: algumas considerações. Paidéia, 22(53), 423-432.

Brown, T. A. (2006). Confirmatory factor analysis for applied research. New York: The Guilford Press.

Buhl, H. M. (2009). My mother: My best friend? Adults relationships with significant others across the lifespan. Journal of Adult Development, 16, 239-249. doi: 10.1007/s10804-009-9070-2

Camargo, V. C. V., Calais, S. L., \& Sartori, M. M. P. (2015). Estresse, depressão e percepção de suporte familiar em estudantes de educação profissionalizante. Estudos de Psicologia (Campinas), 32(4), 595-604. doi: 10.1590/0103-166X2015000400003

Camargo, L. A., Capitão, C. G., \& Filipe, E. M. V. (2014). Mental health, family support and treatment adherence: Associations in the context of HIV/AIDS. Psico-USF, 19(2), 221-232. doi: 10.1590/1413-82712014019002013

Capitão, C. G., Baptista, M. N., Marques, M. A. B., \& Meireles, E. (2013). Inventário de percepção de suporte familiar - IPSF: validade de critério com pacientes em atendimento psicoterápico. Revista Transcultural, 5, 75-93.

Carneiro, T. F. (1983). Entrevista familiar estruturada - EFE: um novo método de avaliação das relações familiares. Em T. F. Carneiro (Ed.) Família: diagnóstico e terapia (pp. 31-52). Rio de Janeiro: Zahar.

Carvalho, S., Pinto-Gouveia, J., Pimentel, P., Maia, D., \& Mota-Pereira, J. (2011). Características psicométricas da versão portuguesa da escala multidimensional de suporte social percebido. Psychologica, 54, 465-492.

Damásio, B. F. (2012). Uso da análise fatorial exploratória em psicologia. Avaliação Psicológica, 11(2), 213-228.

Dancey, C. P., \& Reidy, J. (2006). Estatística sem matemática para psicologia: Usando SPSS para Windows. Porto Alegre: Artmed.

Epstein, N. B., Baldwin, L. M., \& Bishop, D. S., (1983).The McMaster family assessment device. Journal of Marital and Family Therapy, 9(2), 171-80. doi:10.1111/j.1752-0606.1983.tb01497.x 
European Federation of Psychologist's Associations [EFPA]. (2005). Meta-code of ethics (2 ed.). Brussels: European Federation of Psychologist's Associations.

Fingerman, K. L., Miller, L. M., Birditt, K. S., \& Zarit, S. (2009). Giving to the good and the needy: Parental support of grown children. Journal of Marriage and Family, 71(5), 1220-1233. doi: 10.1111/j.1741-3737.2009.00665.x

Gomes, J. O., \& Baptista, M. N. (2013). Funcionamento diferencial dos itens para o inventário de percepção de suporte familiar. Psico PUCRS. 44(4), 490-498.

Gomide, P. I. C. (2003). Estilos parentais e comportamento antissocial. Em A. Del Prette \& Z. Del Prette (Eds.). Habilidades sociais, desenvolvimento e aprendizagem: questões conceituais, avaliação e intervenção (pp. 21-60). Campinas: Alínea.

Gonçalves, M., Farcas, D., \& Martins, M. (2013). Assessment and intervention within social integration income (RSI) in Portugal: professional's perspective. Revista Transcultural, 5(1), 25-35.

Gonçalves, M., Farcas, D., \& Cook, B. (2014). Advocacy for youth mental health in Europe: A policy analysis. International Journal of Clinical Neurosciences and Mental Health, 1(15). Recuperado de http://ijcnmh.arc-publishing.org/editions/6/articles/58

Green, R. G., Kolevzon, M. S., \& Vosler, N. R. (1985). The beavers timberlawn model of family competence and the circumplex model of family adaptability and cohesion: Separate but equal? Family Process, 24(3), 385-398.

Hair, J., Black, W., Babin, B., \& Anderson, R. (2010). Multivariate data analysis: A global perspective. Upper Saddle River: Pearson Education.

Olson, D. H., Portner, J., \& Lavee, Y. (1985). Family adaptability \& cohesion scales - FACES III (research instrument and manual). St. Paul, MN: University of Minnesota, Family Social Science.

Olson, D. H., Russell, C. S., \& Sprenkle, D. H. (1983). Circumplex model of marital and family systems: Theoretical update. Family Process, 22(1), 69-83.

Ordem dos Psicólogos Portugueses (2011). Código Deontológico da Ordem dos Psicólogos Portugueses. Lisboa: Ordem dos Psicólogos Portugueses.

Pardeck, J. T., Brown, C., Christian, B., Schnurbusch, M., Shrum, L., \& Terrell, D. (1991). Family structure and life satisfaction. Family Therapy, 18(1), 11-15.

Parker, G., Tupling, H., \& Brown, L. B. A (1979). Parental Bonding Instrument. British Journal of Medical Psychology, 52(1), 1-10. doi:10.1111/j.2044-8341.1979.tb02487.x

Pasquali, L., \& Araújo, J. M. A. (1986). Questionário de percepção dos pais. Psicologia: Teoria e Pesquisa, 2(1), 56-72.

Reichenheim, M. E., \& Moraes, C. L. (2007). Operacionalização de adaptação transcultural de instrumentos de aferição usados em epidemiologia. Revista de Saúde Pública [on-line]. 41(4), 665-673. Recuperado de http://www.scielo.br/scielo.php?pid=S0034$89102007000400024 \&$ script $=$ sci_arttext

Ross, C. E., \& Mirowsky, J. (2002). Family relationships, social support and subjective life expectancy. Journal of Health and Social Behavior, 43(4), 469-489.

Souza, M. S., Baptista, A. S. D., \& Baptista, M. N. (2010). Relação entre suporte familiar, saúde mental e comportamentos de risco em estudantes universitários. Acta Colombiana de Psicologia, 13(1), 143-154.

recebido em junho de 2015 reformulado em novembro de 2015 aprovado em dezembro de 2015

\section{Sobre os autores}

Marta Gonçalves é psicóloga, doutora pela Universidade de Zurique. Atualmente é pesquisadora na Universidade de Harvard (EUA) e no Instituto Universitário de Lisboa.

Makilim Nunes Baptista é psicólogo, doutor em psiquiatria e psicologia médica pela Universidade Federal de São Paulo. Atualmente é professor do programa de pós-graduação stricto sensu em psicologia da Universidade São Francisco e bolsista produtividade pelo CNPq.

Diana Farcas é psicóloga e doutoranda pelo Instituto Universitário de Lisboa, onde também leciona e desempenha o papel de investigadora assistente em vários projetos de investigação associados ao Centro de Investigação e Intervenção Social. 